\title{
The method of upper and lower solutions to impulsive differential equations with integral boundary conditions
}

\author{
Huihui Pang*, Meng Lu and Chen Cai
}

"Correspondence:

phh2000@163.com

College of Science, China

Agricultural University, Beijing,

100083, P.R. China

\begin{abstract}
This paper considers a second-order impulsive differential equation with integral boundary conditions. Some sufficient conditions for the existence of solutions are proposed by using the method of upper and lower solutions and Leray-Schauder degree theory.
\end{abstract}

Keywords: impulsive differential equation; lower (upper) solution; integral boundary condition; Leray-Schauder degree

\section{Introduction}

The theory of impulsive differential equations describes processes which experience a sudden change of their state at certain moments. Processes with such a character arise naturally and often, especially in phenomena studied in physics, chemical technology, population dynamics, biotechnology, and economics. For an introduction of the basic theory of impulsive differential equations in $R^{n}$, see Lakshmikantham et al. [1], Bainov and Simeonov [2], Samoilenko and Perestyuk [3], and the references therein. The theory of impulsive differential equations has become an important area of investigation in recent years, and it is much richer than the corresponding theory of differential equations (see, for instance, [4-6] and the references therein).

On the other hand, the theory of boundary value problems with integral boundary conditions for ordinary differential equations arises in different areas of applied mathematics and physics. For example, heat conduction, chemical engineering, underground water flow, thermo-elasticity, and plasma physics can be reduced to nonlocal problems with integral boundary conditions. For boundary value problems with integral boundary conditions and comments on their importance, we refer the reader to the papers in Gallardo [7-9] and the references therein. For more information about the general theory of integral equations and their relation with boundary value problems, we refer to the book of Corduneanu [10] and Agarwal and O'Regan [11].

Recently, some well-known works, such as Hao et al. [12], Zhang et al. [13] and Ding and Wang [14], deal with impulsive differential equations with integral boundary conditions. However, most of these results are obtained by using the fixed point theorem in cones. It is well known that the method of upper and lower solutions is a powerful tool for proving the existence results for a large class of boundary value problems, see [15-17].

@2014 Pang et al.; licensee Springer. This is an Open Access article distributed under the terms of the Creative Commons Attribution License (http://creativecommons.org/licenses/by/2.0), which permits unrestricted use, distribution, and reproduction in any medium, provided the original work is properly cited. 
In [17], Shen and Wang applied the method of upper and lower solutions to solve impulsive differential equations with nonlinear boundary conditions as follows:

$$
\left\{\begin{array}{l}
x^{\prime \prime}(t)=f\left(t, x(t), x^{\prime}(t)\right), \quad t \in J, t \neq t_{k}, \\
\Delta x\left(t_{k}\right)=I_{k}\left(x\left(t_{k}\right)\right), \quad k=1,2, \ldots, p, \\
\Delta x^{\prime}\left(t_{k}\right)=J_{k}\left(x\left(t_{k}\right), x^{\prime}\left(t_{k}\right)\right), \quad k=1,2, \ldots, p, \\
g\left(x(0), x^{\prime}(0)\right)=0, \quad h\left(x(1), x^{\prime}(1)\right)=0 .
\end{array}\right.
$$

Motivated by the works mentioned above, in this paper, we shall employ the method of upper and lower solutions together with Leray-Schauder degree theory to study the existence of a solution of the impulsive BVP

$$
\left\{\begin{array}{l}
x^{\prime \prime}(t)+f\left(t, x(t), x^{\prime}(t)\right)=0, \quad t \in J^{*}, \\
\Delta x\left(t_{k}\right)=I_{k}\left(x\left(t_{k}\right)\right), \quad k=1,2, \ldots, p, \\
\Delta x^{\prime}\left(t_{k}\right)=J_{k}\left(x\left(t_{k}\right), x^{\prime}\left(t_{k}\right)\right), \quad k=1,2, \ldots, p, \\
x(0)=x(1)=\int_{0}^{1} g(s) x(s) d s,
\end{array}\right.
$$

where $J=[0,1], J^{*}=J \backslash\left\{t_{1}, t_{2}, \ldots, t_{p}\right\}, f: J \times R^{2} \rightarrow R$ is continuous, $I_{k}, J_{k} \in C(R)$ for $1 \leq k \leq p$, $g \in L^{1}[0,1]$ is nonnegative, $0=t_{0}<t_{1}<t_{2}<\cdots<t_{p}<t_{p+1}=1, \Delta x\left(t_{k}\right)=x\left(t_{k}^{+}\right)-x\left(t_{k}^{-}\right)$denotes the jump of $x(t)$ at $t=t_{k}, x\left(t_{k}^{+}\right)$and $x\left(t_{k}^{-}\right)$represent the right and left limits of $x(t)$ at $t=t_{k}$, respectively, $\Delta x^{\prime}\left(t_{k}\right)$ has a similar meaning for $x^{\prime}(t)$.

\section{Preliminaries}

Define $P C(J)=\left\{x: J \rightarrow R \mid x \in C\left(J^{*}\right), x\left(t_{i}^{+}\right)\right.$and $x\left(t_{i}^{-}\right)$exist, and $\left.x\left(t_{i}^{-}\right)=x\left(t_{i}\right), i=1,2, \ldots, p\right\}$. $P C^{1}(J)=\left\{x \in P C(J):\left.x\right|_{\left(t_{i}, t_{i+1}\right)} \in C^{1}\left(t_{i}, t_{i+1}\right), x^{\prime}\left(t_{i}^{+}\right)\right.$and $x^{\prime}\left(t_{i}^{-}\right)$exist, and $x^{\prime}\left(t_{i}^{-}\right)=x^{\prime}\left(t_{i}\right), i=$ $1,2, \ldots, p\}$. Note that $P C(J)$ and $P C^{1}(J)$ are Banach spaces with the respective norms

$$
\|x\|_{\infty}=\sup \{|x(t)|: t \in J\}, \quad\|x\|=\max \left\{\|x\|_{\infty},\left\|x^{\prime}\right\|_{\infty}\right\}
$$

A function $x \in E=P C^{1}(J) \cap C^{2}\left(J^{*}\right)$ is called a solution of (1.1) if it satisfies the differential equation

$$
x^{\prime \prime}(t)+f\left(t, x(t), x^{\prime}(t)\right)=0, \quad t \in J^{*},
$$

the impulsive conditions $\Delta x\left(t_{k}\right)=I_{k}\left(x\left(t_{k}\right)\right), \Delta x^{\prime}\left(t_{k}\right)=J_{k}\left(x\left(t_{k}\right), x^{\prime}\left(t_{k}\right)\right)$ and the boundary conditions $x(0)=x(1)=\int_{0}^{1} g(s) x(s) d s$.

Definition 2.1 The function $\alpha \in E$ is said to be a lower solution for boundary value problem (1.1) if

$$
\begin{aligned}
& \alpha^{\prime \prime}(t)+f\left(t, \alpha(t), \alpha^{\prime}(t)\right) \geq 0, \quad t \in J^{*}, \\
& \Delta \alpha\left(t_{k}\right)=I_{k}\left(\alpha\left(t_{k}\right)\right), \quad \Delta \alpha^{\prime}\left(t_{k}\right)=J_{k}\left(\alpha\left(t_{k}\right), \alpha^{\prime}\left(t_{k}\right)\right), \quad k=1,2, \ldots, p, \\
& \alpha(0) \leq \int_{0}^{1} g(s) \alpha(s) d s, \quad \alpha(1) \leq \int_{0}^{1} g(s) \alpha(s) d s .
\end{aligned}
$$


The function $\beta \in E$ is said to be an upper solution for boundary value problem (1.1) if

$$
\begin{aligned}
& \beta^{\prime \prime}(t)+f\left(t, \beta(t), \beta^{\prime}(t)\right) \leq 0, \quad t \in J^{*}, \\
& \Delta \beta\left(t_{k}\right)=I_{k}\left(\beta\left(t_{k}\right)\right), \quad \Delta \beta^{\prime}\left(t_{k}\right)=J_{k}\left(\beta\left(t_{k}\right), \beta^{\prime}\left(t_{k}\right)\right), \quad k=1,2, \ldots, p, \\
& \beta(0) \geq \int_{0}^{1} g(s) \beta(s) d s, \quad \beta(1) \geq \int_{0}^{1} g(s) \beta(s) d s .
\end{aligned}
$$

For $\alpha, \beta \in E$, we write $\alpha \leq \beta$ if $\alpha(t) \leq \beta(t)$ for all $t \in J$.

Definition 2.2 Let $\alpha, \beta \in E$ be such that $\alpha \leq \beta$ on $J$. We say that $f$ satisfies the Nagumo condition relative to $\alpha, \beta$ if for

$$
\omega=\max _{1 \leq k \leq p}\left\{\frac{\left|\alpha\left(t_{k+1}\right)-\beta\left(t_{k}^{+}\right)\right|}{t_{k+1}-t_{k}}, \frac{\left|\beta\left(t_{k+1}\right)-\alpha\left(t_{k}^{+}\right)\right|}{t_{k+1}-t_{k}}\right\},
$$

there exists a constant $D$ such that

$$
D>\max \left\{\omega,\left\|\alpha^{\prime}\right\|_{\infty},\left\|\beta^{\prime}\right\|_{\infty}\right\},
$$

a continuous function $\Psi:[0, \infty) \rightarrow(0, \infty)$, and constants $A \geq 0, B \geq 0$ such that

$$
|f(t, x, y)| \leq A|y| \Psi(|y|)+B, \quad t \in J, \alpha \leq x \leq \beta, y \in R
$$

and

$$
\int_{\omega}^{D} \frac{1}{\Psi(s)} d s>A\left[\max _{t \in J} \beta(t)-\min _{t \in J} \alpha(t)\right]+B \max _{s \geq \omega} \Psi^{-1}(s) .
$$

In addition, we assume that the following conditions hold:

$\left(\mathrm{H}_{1}\right) f$ satisfies the Nagumo condition relative to $\alpha, \beta$;

$\left(\mathrm{H}_{2}\right) I_{k}, J_{k} \in C(R, R) . J_{k}(y, z)$ is nondecreasing in $z \in[-D, D]$ for all $1 \leq k \leq p$;

$\left(\mathrm{H}_{3}\right) \quad 0<\int_{0}^{1} g(s) d s<1$.

We consider the modified problem

$$
\begin{aligned}
& x^{\prime \prime}(t)+F\left(t, x(t), \frac{d}{d t} m_{\alpha, \beta}(t, x)\right)=0, \quad t \in J^{*}, \\
& \Delta x\left(t_{k}\right)=I_{k}\left(m_{\alpha, \beta}\left(t_{k}, x\left(t_{k}\right)\right)\right), \quad k=1,2, \ldots, p, \\
& \Delta x^{\prime}\left(t_{k}\right)=J_{k}\left(m_{\alpha, \beta}\left(t_{k}, x\left(t_{k}\right)\right), n\left(t_{k}, x^{\prime}\left(t_{k}\right)\right)\right), \quad k=1,2, \ldots, p, \\
& x(0)=x(1)=\int_{0}^{1} g(s) x(s) d s,
\end{aligned}
$$

where

$$
F(t, x, y)=f\left(t, m_{\alpha, \beta}(t, x), n(t, y)\right)+\frac{m_{\alpha, \beta}(t, x)-x}{1+\left(x-m_{\alpha, \beta}(t, x)\right)^{2}}
$$


and

$$
\begin{aligned}
& m_{\alpha, \beta}(t, x)=\max \{\alpha(t), \min \{x, \beta(t)\}\}, \\
& n(t, x)=\max \{-D, \min \{x, D\}\} .
\end{aligned}
$$

One can find the next result, with its proof, in [18].

Lemma 2.1 For each $x \in E$, the following two properties hold:

(i) $\frac{d}{d t} m_{\alpha, \beta}(t, x)$ exists for a.e. $t \in J^{*}$;

(ii) if $x, x_{m} \in E$ and $x_{m} \rightarrow x$ in $E$, then $\frac{d}{d t} m_{\alpha, \beta}\left(t, x_{m}(t)\right) \rightarrow \frac{d}{d t} m_{\alpha, \beta}(t, x(t))$ for a.e. $t \in J^{*}$.

Lemma 2.2 For any $v(t) \in C(J)$, the following boundary value problem

$$
\left\{\begin{array}{l}
x^{\prime \prime}(t)+v(t)=0, \quad t \in J^{*}, \\
\Delta x\left(t_{k}\right)=I_{k}\left(x\left(t_{k}\right)\right), \quad k=1,2, \ldots, p, \\
\Delta x^{\prime}\left(t_{k}\right)=J_{k}\left(x\left(t_{k}\right), x^{\prime}\left(t_{k}\right)\right), \quad k=1,2, \ldots, p, \\
x(0)=x(1)=\int_{0}^{1} g(s) x(s) d s
\end{array}\right.
$$

has a unique solution as follows:

$$
\begin{aligned}
x(t)= & \int_{0}^{1} H(t, s) v(s) d s+\sum_{0<t_{k}<t}\left[I_{k}\left(x\left(t_{k}\right)\right)+\left(t-t_{k}\right) J_{k}\left(x\left(t_{k}\right), x^{\prime}\left(t_{k}\right)\right)\right] \\
& -t \sum_{k=1}^{p}\left[I_{k}\left(x\left(t_{k}\right)\right)+\left(1-t_{k}\right) J_{k}\left(x\left(t_{k}\right), x^{\prime}\left(t_{k}\right)\right)\right] \\
& +\frac{1}{1-\mu} \int_{0}^{1} \sum_{0<t_{k}<s}\left[I_{k}\left(x\left(t_{k}\right)\right)+\left(s-t_{k}\right) J_{k}\left(x\left(t_{k}\right), x^{\prime}\left(t_{k}\right)\right)\right] d s \\
& -\frac{v}{1-\mu} \sum_{k=1}^{p}\left[I_{k}\left(x\left(t_{k}\right)\right)+\left(1-t_{k}\right) J_{k}\left(x\left(t_{k}\right), x^{\prime}\left(t_{k}\right)\right)\right],
\end{aligned}
$$

where

$$
\begin{aligned}
& H(t, s)=G(t, s)+\frac{1}{1-\mu} \int_{0}^{1} G(s, \tau) g(\tau) d \tau, \\
& G(t, s)= \begin{cases}t(1-s), & 0 \leq t \leq s \leq 1, \\
s(1-t), & 0 \leq s \leq t \leq 1,\end{cases}
\end{aligned}
$$

and

$$
\mu=\int_{0}^{1} g(s) d s, \quad v=\int_{0}^{1} s g(s) d s .
$$

Lemma 2.3 If $x$ is a solution of $B V P(2.1), \alpha(t)$ and $\beta(t)$ are lower and upper solutions of (1.1), respectively, $\alpha \leq \beta$, and

$$
I_{k}\left(\alpha\left(t_{k}\right)\right) \leq I_{k}(x) \leq I_{k}\left(\beta\left(t_{k}\right)\right), \quad k=1, \ldots, p,
$$


for

$$
\alpha\left(t_{k}\right) \leq x \leq \beta\left(t_{k}\right),
$$

then

$$
\alpha(t) \leq x(t) \leq \beta(t), \quad t \in[0,1] .
$$

Proof Denote $y(t)=x(t)-\beta(t)$, we will only see that $x(t) \leq \beta(t)$ for every $t \in J$. An analogous reasoning shows that $x(t) \geq \alpha(t)$ for all $t \in J$. Otherwise, if $x(t) \leq \beta(t), t \in[0,1]$, does not hold, then $\sup _{0 \leq t \leq 1}(x(t)-\beta(t))>0$, there are three cases.

Case 1. Suppose that $\max _{0 \leq t \leq 1} y(t)=\sup _{0 \leq t \leq 1}(x(t)-\beta(t))=y(0)$, or $\max _{0 \leq t \leq 1} y(t)=y(1)$, we only see that $\max _{0 \leq t \leq 1} y(t)=y(0)$. Easily, it holds that $y(0)>0$. From Definition 2.1 and $\left(\mathrm{H}_{3}\right)$, we have

$$
\begin{aligned}
y(0) & =x(0)-\beta(0) \leq \int_{0}^{1} g(s)(x(s)-\beta(s)) d s \\
& \leq \max _{s \in[0,1]}(x(s)-\beta(s)) \int_{0}^{1} g(s) d s \\
& <\max _{s \in[0,1]}(x(s)-\beta(s))=\max _{s \in[0,1]} y(s),
\end{aligned}
$$

which is a contradiction.

Case 2. Suppose that there exist $k \in\{0,1, \ldots, p\}$ and $\tau \in\left(t_{k}, t_{k}+1\right)$ such that

$$
\sup _{t \in\left(t_{k}, t_{k}+1\right]} y(t)=y(\tau)=x(\tau)-\beta(\tau)>0 .
$$

Then $y^{\prime}(\tau)=0$ and $y^{\prime \prime}(\tau) \leq 0$. On the other hand,

$$
\begin{aligned}
y^{\prime \prime}(\tau) \geq & -F\left(\tau, x(\tau), \frac{d}{d t} m_{\alpha, \beta}(\tau, x)\right)+f\left(\tau, \beta(\tau), \beta^{\prime}(\tau)\right) \\
= & -f\left(\tau, m(\tau, x(\tau)), \frac{d}{d t} m_{\alpha, \beta}(\tau, x)\right)-\frac{m_{\alpha, \beta}(\tau, x(\tau))-x(\tau)}{1+\left(x(\tau)-m_{\alpha, \beta}(\tau, x(\tau))\right)^{2}} \\
& +f\left(\tau, \beta(\tau), \beta^{\prime}(\tau)\right) \\
= & -f\left(\tau, \beta(\tau), \beta^{\prime}(\tau)\right)-\frac{m_{\alpha, \beta}(\tau, x(\tau))-x(\tau)}{1+\left(x(\tau)-m_{\alpha, \beta}(\tau, x(\tau))\right)^{2}}+f\left(\tau, \beta(\tau), \beta^{\prime}(\tau)\right) \\
= & \frac{y(\tau)}{1+y(\tau)^{2}}>0,
\end{aligned}
$$

which is a contradiction. Hence the function $y$ cannot have any positive maximum interior to the interval $\left(t_{k}, t_{k}+1\right)$ for $k=1,2, \ldots, p$.

Case 3. According to Case 2, if $\sup _{0 \leq t \leq 1} y(t)>0$, then $\sup _{0 \leq t \leq 1} y(t)=y\left(t_{k}^{+}\right)$, or $\sup _{0 \leq t \leq 1} y(t)=y\left(t_{k}^{-}\right)=y\left(t_{k}\right)$, we only prove that $\sup _{0 \leq t \leq 1} y(t)=y\left(t_{k}^{-}\right), k=1,2, \ldots, p$. Suppose that $\sup _{0 \leq t \leq 1} y(t)=y\left(t_{1}^{-}\right)$, easily, $y^{\prime}\left(t_{1}\right)=y^{\prime}\left(t_{1}^{-}\right) \geq 0$. 
From (2.1) and (2.3), we have

$$
\begin{aligned}
x\left(t_{1}^{+}\right)-x\left(t_{1}\right) & =x\left(t_{1}^{+}\right)-x\left(t_{1}^{-}\right)=I_{1}\left(m_{\alpha, \beta}\left(t_{1}, x\left(t_{1}\right)\right)\right) \\
& =I_{1}\left(\beta\left(t_{1}\right)\right)=\beta\left(t_{1}^{+}\right)-\beta\left(t_{1}^{-}\right)=\beta\left(t_{1}^{+}\right)-\beta\left(t_{1}\right), \\
x^{\prime}\left(t_{1}^{+}\right)-x^{\prime}\left(t_{1}\right) & =x^{\prime}\left(t_{1}^{+}\right)-x^{\prime}\left(t_{1}^{-}\right)=J_{1}\left(m_{\alpha, \beta}\left(t_{1}, x\left(t_{1}\right)\right), n\left(t_{1}, x^{\prime}\left(t_{1}\right)\right)\right) \\
& \geq J_{1}\left(\beta\left(t_{1}\right), \beta^{\prime}\left(t_{1}\right)\right) \geq \beta^{\prime}\left(t_{1}^{+}\right)-\beta^{\prime}\left(t_{1}^{-}\right) .
\end{aligned}
$$

Hence

$$
\begin{aligned}
& y\left(t_{1}^{+}\right)=x\left(t_{1}^{+}\right)-\beta\left(t_{1}^{+}\right)=x\left(t_{1}\right)-\beta\left(t_{1}\right)>0, \\
& y^{\prime}\left(t_{1}^{+}\right)=x^{\prime}\left(t_{1}^{+}\right)-\beta^{\prime}\left(t_{1}^{+}\right) \geq x^{\prime}\left(t_{1}\right)-\beta^{\prime}\left(t_{1}\right) \geq 0 .
\end{aligned}
$$

Suppose that $y^{\prime}\left(t_{1}^{+}\right)=0$ and $y$ is nonincreasing on some interval $\left(t_{1}, t_{1}+\gamma\right) \subset\left(t_{1}, t_{2}\right)$, where $\gamma>0$ is sufficiently small such that $y(t)>0$ on $t \in\left(t_{1}, t_{1}+\gamma\right)$. For $t \in\left(t_{1}, t_{1}+\gamma\right)$,

$$
\begin{aligned}
x^{\prime \prime}(t)-\beta^{\prime \prime}(t) & \geq-f\left(t, \beta(t), \frac{d}{d t} m_{\alpha, \beta}(t, x)\right)-\frac{m_{\alpha, \beta}(t, x)-x}{1+\left(x-m_{\alpha, \beta}(t, x)\right)^{2}}+f\left(t, \beta(t), \beta^{\prime}(t)\right) \\
& =\frac{m_{\alpha, \beta}(t, x)-x}{1+\left(x-m_{\alpha, \beta}(t, x)\right)^{2}}>0,
\end{aligned}
$$

which contradicts the assumption of monotonicity of $y$. Thus, we obtain

$$
\begin{aligned}
& 0<y\left(t_{1}^{+}\right)<y\left(t_{2}\right)=y\left(t_{2}^{-}\right), \\
& y^{\prime}\left(t_{2}\right)=y^{\prime}\left(t_{2}^{-}\right) \geq 0 .
\end{aligned}
$$

We use the preceding procedure and deduce by induction that

$$
y\left(t_{k}\right)>0, \quad y^{\prime}\left(t_{k}\right) \geq 0, \quad k=1,2, \ldots, p+1,
$$

which contradicts that $y(1)$ cannot be the maximum point. By the same analysis, we can get that $y\left(t_{k}\right)>0, k=2,3, \ldots, p$, cannot hold.

Lemma 2.4 $-D \leq x^{\prime}(t) \leq D$ on $J$, where $x(t)$ is the solution of $(2.1)$.

Proof Here we only show $x^{\prime}(t) \leq D$. Suppose that there exists $\rho \in\left(t_{k}, t_{k+1}\right)$ with $x^{\prime}(\rho)=$ $\frac{x\left(t_{k+1}\right)-x\left(t_{k}\right)}{t_{k+1}-t_{k}}, k \in\{1,2, \ldots, p\}$, and as a result,

$$
-D<-\omega \leq \frac{\alpha\left(t_{k+1}\right)-\beta\left(t_{k}\right)}{t_{k+1}-t_{k}} \leq x^{\prime}(\rho) \leq \frac{\beta\left(t_{k+1}\right)-\alpha\left(t_{k}\right)}{t_{k+1}-t_{k}} \leq \omega<D .
$$

Therefore, there exist $r_{1}, r_{2} \in\left(t_{k}, t_{k+1}\right)$ such that $x^{\prime}\left(r_{1}\right)=\omega, x^{\prime}\left(r_{2}\right)=D$ and either

$$
\omega \leq x^{\prime}(t) \leq D, \quad t \in\left(r_{1}, r_{2}\right)
$$

or

$$
\omega \leq x^{\prime}(t) \leq D, \quad t \in\left(r_{2}, r_{1}\right)
$$


We only consider the first case, since the other case can be handled similarly. It follows from the assumption that

$$
x^{\prime \prime}(t) \leq A x^{\prime}(t) \Psi\left(x^{\prime}(t)\right)+B \quad \text { for } t \in\left(r_{1}, r_{2}\right) .
$$

This implies that

$$
\begin{aligned}
\int_{r_{1}}^{r_{2}} \frac{x^{\prime \prime}(t)}{\Psi\left(x^{\prime}(t)\right)} d t & \leq A \int_{r_{1}}^{r_{2}} x^{\prime}(t) d t+B \int_{r_{1}}^{r_{2}} \Psi^{-1}\left(x^{\prime}(t)\right) d t \\
& =A\left(x\left(r_{2}\right)-x\left(r_{1}\right)\right)+B \int_{r_{1}}^{r_{2}} \Psi^{-1}\left(x^{\prime}(t)\right) d t
\end{aligned}
$$

which yields

$$
\begin{aligned}
\int_{\omega}^{D} \frac{d s}{\Psi(s)} & =\int_{x^{\prime}\left(r_{1}\right)}^{x^{\prime}\left(r_{2}\right)} \frac{d s}{\Psi(s)} \leq A\left(x\left(r_{2}\right)-x\left(r_{1}\right)\right)+B\left(r_{2}-r_{1}\right) \max _{s \geq \omega} \Psi^{-1}(s) \\
& \leq A\left(\max _{t \in J} \beta(t)-\min _{t \in J} \alpha(t)\right)+B \max _{s \geq \omega} \Psi^{-1}(s) .
\end{aligned}
$$

This, obviously, contradicts the choice of $D$. The proof is complete.

Lemma 2.5 (Schauder's fixed point theorem) Let $K$ be a convex subset of a normed linear space E. Each continuous, compact map $L: K \rightarrow K$ has a fixed point.

\section{Existence results}

Theorem 3.1 Suppose that conditions $\left(\mathrm{H}_{1}\right)-\left(\mathrm{H}_{3}\right)$ hold. Then BVP (1.1) has at least one solution $x \in E \cap C^{2}\left(J^{*}\right)$ such that

$$
\alpha(t) \leq x(t) \leq \beta(t), \quad-D \leq x^{\prime}(t) \leq D, \quad t \in[0,1] .
$$

Proof Solving (2.1) is equivalent to finding $x \in E$ which satisfies

$$
\begin{aligned}
x(t)= & \int_{0}^{1} H(t, s) F\left(s, x(s), \frac{d}{d s} m_{\alpha, \beta}(s, x)\right) d s+\sum_{0<t_{k}<t}\left[I_{k}^{*}\left(x\left(t_{k}\right)\right)+\left(t-t_{k}\right) J_{k}^{*}\left(x\left(t_{k}\right), x^{\prime}\left(t_{k}\right)\right)\right] \\
& -t \sum_{k=1}^{p}\left[I_{k}^{*}\left(x\left(t_{k}\right)\right)+\left(1-t_{k}\right) J_{k}^{*}\left(x\left(t_{k}\right), x^{\prime}\left(t_{k}\right)\right)\right]+\frac{1}{1-\mu} \int_{0}^{1} \sum_{0<t_{k}<s}\left[I_{k}^{*}\left(x\left(t_{k}\right)\right)\right. \\
& \left.+\left(s-t_{k}\right) J_{k}^{*}\left(x\left(t_{k}\right), x^{\prime}\left(t_{k}\right)\right)\right] d s-\frac{v}{1-\mu} \sum_{k=1}^{p}\left[I_{k}^{*}\left(x\left(t_{k}\right)\right)+\left(1-t_{k}\right) J_{k}^{*}\left(x\left(t_{k}\right), x^{\prime}\left(t_{k}\right)\right)\right],
\end{aligned}
$$

where $I_{k}^{*}\left(x\left(t_{k}\right)\right)=I_{k}\left(m_{\alpha, \beta}\left(t_{k}, x\left(t_{k}\right)\right)\right), J_{k}^{*}\left(x\left(t_{k}\right), x^{\prime}\left(t_{k}\right)\right)=J_{k}\left(m_{\alpha, \beta}\left(t_{k}, x\left(t_{k}\right)\right), n\left(t_{k}, x^{\prime}\left(t_{k}\right)\right)\right)$.

Now, define the following operator $T: E \rightarrow E$ by

$$
\begin{aligned}
(T x)(t)= & \int_{0}^{1} H(t, s) F\left(s, x(s), \frac{d}{d s} m_{\alpha, \beta}(s, x)\right) d s \\
& +\sum_{0<t_{k}<t}\left[I_{k}^{*}\left(x\left(t_{k}\right)\right)+\left(t-t_{k}\right) J_{k}^{*}\left(x\left(t_{k}\right), x^{\prime}\left(t_{k}\right)\right)\right]
\end{aligned}
$$




$$
\begin{aligned}
& -t \sum_{k=1}^{p}\left[I_{k}^{*}\left(x\left(t_{k}\right)\right)+\left(1-t_{k}\right) J_{k}^{*}\left(x\left(t_{k}\right), x^{\prime}\left(t_{k}\right)\right)\right]+\frac{1}{1-\mu} \int_{0}^{1} \sum_{0<t_{k}<s}\left[I_{k}^{*}\left(x\left(t_{k}\right)\right)\right. \\
& \left.+\left(s-t_{k}\right) J_{k}^{*}\left(x\left(t_{k}\right), x^{\prime}\left(t_{k}\right)\right)\right] d s-\frac{v}{1-\mu} \sum_{k=1}^{p}\left[I_{k}^{*}\left(x\left(t_{k}\right)\right)+\left(1-t_{k}\right) J_{k}^{*}\left(x\left(t_{k}\right), x^{\prime}\left(t_{k}\right)\right)\right] .
\end{aligned}
$$

It is obvious that $T: E \rightarrow E$ is completely continuous.

By the Schauder fixed point theorem, we can easily obtain that $T$ has a fixed point $x \in E$, which is a solution of BVP (2.1). And by Lemma 2.3 and Lemma 2.4, we know that $\alpha(t) \leq$ $x(t) \leq \beta(t),-D \leq x^{\prime}(t) \leq D$, then BVP (2.1) becomes BVP (1.1), therefore $x(t)$ is a solution of BVP (1.1). The proof is complete.

Theorem 3.2 Suppose that conditions $\left(\mathrm{H}_{1}\right)-\left(\mathrm{H}_{3}\right)$ hold. Assume that there exist two lower solutions $\alpha_{1}$ and $\alpha_{2}$ and two upper solutions $\beta_{1}$ and $\beta_{2}$ for problem (1.1), satisfying the following:

(i) $\alpha_{1} \leq \alpha_{2} \leq \beta_{2}$;

(ii) $\alpha_{1} \leq \beta_{1} \leq \beta_{2}$;

(iii) $\alpha_{2} \nless \beta_{1}$, which means that there exists $t \in[0,1]$ such that $\alpha_{2}>\beta_{1}$;

(iv) if $x$ is a solution of $(1.1)$ with $x \geq \alpha_{2}$, then $x>\alpha_{2}$ on $(0,1)$;

(v) if $x$ is a solution of (1.1) with $x \leq \beta_{1}$, then $x<\beta_{1}$ on $(0,1)$.

Iff satisfies the Nagumo condition with respect to $\alpha_{1}, \beta_{2}$, then problem (1.1) has at least three solutions $x_{1}, x_{2}$ and $x_{3}$ satisfying

$$
\alpha_{1} \leq x_{1} \leq \beta_{1}, \quad \alpha_{2} \leq x_{2} \leq \beta_{2}, \quad x_{3} \not \leq \beta_{1}, \quad x_{3} \nsupseteq \alpha_{2} .
$$

Proof We consider the following modified problem:

$$
\begin{aligned}
& x^{\prime \prime}(t)+F\left(t, x(t), \frac{d}{d t} m_{\alpha_{1}, \beta_{2}}(t, x)\right)=0, \quad t \in J^{*}, \\
& \Delta x\left(t_{k}\right)=\bar{I}_{k}\left(x\left(t_{k}\right)\right), \quad k=1,2, \ldots, p, \\
& \Delta x^{\prime}\left(t_{k}\right)=\bar{J}_{k}\left(x\left(t_{k}\right), x^{\prime}\left(t_{k}\right)\right), \quad k=1,2, \ldots, p, \\
& x(0)=x(1)=\int_{0}^{1} g(s) x(s) d s,
\end{aligned}
$$

where $\bar{I}_{k}\left(x\left(t_{k}\right)\right)=I_{k}\left(m_{\alpha_{1}, \beta_{2}}\left(t_{k}, x\left(t_{k}\right)\right)\right), \bar{J}_{k}\left(x\left(t_{k}\right), x^{\prime}\left(t_{k}\right)\right)=J_{k}\left(m_{\alpha_{1}, \beta_{2}}\left(t_{k}, x\left(t_{k}\right)\right), n\left(t_{k}, x^{\prime}\left(t_{k}\right)\right)\right)$.

Now define the following operator $\bar{T}: E \rightarrow E$ by

$$
\begin{aligned}
(\bar{T} x)(t)= & \int_{0}^{1} H(t, s) F\left(s, x(s), \frac{d}{d s} m_{\alpha_{1}, \beta_{2}}(s, x)\right) d s \\
& +\sum_{0<t_{k}<t}\left[\bar{I}_{k}\left(x\left(t_{k}\right)\right)+\left(t-t_{k}\right) \bar{J}_{k}\left(x\left(t_{k}\right), x^{\prime}\left(t_{k}\right)\right)\right] \\
& -t \sum_{k=1}^{p}\left[\bar{I}_{k}\left(x\left(t_{k}\right)\right)+\left(1-t_{k}\right) \bar{J}_{k}\left(x\left(t_{k}\right), x^{\prime}\left(t_{k}\right)\right)\right]+\frac{1}{1-\mu} \int_{0}^{1} \sum_{0<t_{k}<s}\left[\bar{I}_{k}\left(x\left(t_{k}\right)\right)\right. \\
& \left.+\left(s-t_{k}\right) \bar{J}_{k}\left(x\left(t_{k}\right), x^{\prime}\left(t_{k}\right)\right)\right] d s-\frac{v}{1-\mu} \sum_{k=1}^{p}\left[\bar{I}_{k}\left(x\left(t_{k}\right)\right)+\left(1-t_{k}\right) \bar{J}_{k}\left(x\left(t_{k}\right), x^{\prime}\left(t_{k}\right)\right)\right] .
\end{aligned}
$$


Since the functions $m_{\alpha_{1}, \beta_{2}}$ and $n$ are continuous and bounded, we obtain that there exists $M_{F}>0$ such that

$$
\left|F\left(t, x(t), x^{\prime}(t)\right)\right| \leq M_{F}
$$

Let

$$
M=\frac{2 M_{F}}{1-\mu}+\frac{3+v-2 \mu}{1-\mu} \sum_{k=1}^{p}\left[\max _{x \in[-d, d]}\left|I_{k}(x)\right|+\max _{x \in[-d, d], y \in[-D, D]}\left|J_{k}(x, y)\right|\right],
$$

where $d=1+\max _{t \in J}\left\{\left\|\alpha_{1}\right\|_{\infty},\left\|\beta_{2}\right\|_{\infty}\right\}$ and

$$
\Omega=\left\{x \in E,\|x\|_{\infty} \leq M\right\}
$$

It is standard that $\bar{T}: E \rightarrow E$ is completely continuous. It is immediate from the argument above that $\bar{T}(\bar{\Omega}) \subset \Omega$.

Thus,

$$
\operatorname{deg}(I-\bar{T}, \Omega, 0)=1
$$

Let

$$
\Omega_{\alpha_{2}}=\left\{x \in \Omega: x>\alpha_{2} \text { on }(0,1)\right\}, \quad \Omega^{\beta_{1}}=\left\{x \in \Omega: x<\beta_{1} \text { on }(0,1)\right\} .
$$

Since $\alpha_{2} \not \leq \beta_{1}, \alpha_{2}>-\bar{M}, \beta_{1}<\bar{M}$ (i.e., choose $\bar{M}$ such that $\left\|\alpha_{2}\right\|_{\infty},\left\|\beta_{1}\right\|_{\infty}<M$ ). It follows that $\Omega^{\beta_{1}} \neq \emptyset \neq \Omega_{\alpha_{2}}, \Omega^{\beta_{1}} \cap \Omega_{\alpha_{2}}=\emptyset$, and $\Omega \backslash\left(\overline{\Omega^{\beta_{1}} \cup \Omega_{\alpha_{2}}}\right) \neq \emptyset$. By assumptions (iv) and (v), there are no solutions in $\partial \Omega^{\beta_{1}} \cup \partial \Omega_{\alpha_{2}}$. Thus,

$$
\operatorname{deg}(I-\bar{T}, \Omega, 0)=\operatorname{deg}\left(I-\bar{T}, \Omega \backslash\left(\overline{\Omega^{\beta_{1}} \cup \Omega_{\alpha_{2}}}\right), 0\right)+\operatorname{deg}\left(I-\bar{T}, \Omega^{\beta_{1}}, 0\right)+\operatorname{deg}\left(I-\bar{T}, \Omega_{\alpha_{2}}, 0\right) .
$$

We show that $\operatorname{deg}\left(I-\bar{T}, \Omega^{\beta_{1}}, 0\right)=\operatorname{deg}\left(I-\bar{T}, \Omega_{\alpha_{2}}, 0\right)=1$, then

$$
\operatorname{deg}\left(I-\bar{T}, \Omega \backslash\left(\overline{\Omega^{\beta_{1}} \cup \Omega_{\alpha_{2}}}\right), 0\right)=-1
$$

and there are solutions in $\overline{\Omega^{\beta_{1}} \cup \Omega_{\alpha_{2}}}, \Omega^{\beta_{1}}, \Omega_{\alpha_{2}}$, as required.

We now show $\operatorname{deg}\left(I-\bar{T}, \Omega_{\alpha_{2}}, 0\right)=1$. The proof that $\operatorname{deg}\left(I-\bar{T}, \Omega^{\beta_{1}}, 0\right)=1$ is similar and hence omitted. We define $I-W$, the extension to $\bar{\Omega}$ of the restriction of $I-\bar{T}$ to $\bar{\Omega}_{\alpha_{2}}$ as follows.

Let

$$
w(t, x, y)=f\left(t, m_{\alpha_{2}, \beta_{2}}(t, x), n(t, y)\right)+\frac{m_{\alpha_{2}, \beta_{2}}(t, x)-x}{1+\left(x-m_{\alpha_{2}, \beta_{2}}(t, x)\right)^{2}} .
$$

Thus, $w$ is a continuous function on $[0,1] \times R^{2}$ and satisfies

$$
\begin{aligned}
& |w(t, x, y)| \leq A|y| \Psi(|y|)+B \text { for }|y|<D, \\
& |w(t, x, y)| \leq M_{1} \quad \text { for }(t, x, y) \in[0,1] \times R^{2}
\end{aligned}
$$

for some constants $M_{1}$. Moreover, we may choose $M_{1}$ so that $\left\|\alpha_{2}\right\|_{\infty},\left\|\beta_{1}\right\|_{\infty}<M_{1}$. 
Consider the following problem:

$$
\begin{aligned}
& x^{\prime \prime}(t)+w\left(t, x(t), \frac{d}{d t} m_{\alpha_{2}, \beta_{2}}(t, x)\right)=0, \quad t \in J^{*}, \\
& \Delta x\left(t_{k}\right)=\tilde{I}_{k}\left(x\left(t_{k}\right)\right), \quad k=1,2, \ldots, p, \\
& \Delta x^{\prime}\left(t_{k}\right)=\tilde{J}_{k}\left(x\left(t_{k}\right), x^{\prime}\left(t_{k}\right)\right), \quad k=1,2, \ldots, p, \\
& x(0)=x(1)=\int_{0}^{1} g(s) x(s) d s,
\end{aligned}
$$

where $\tilde{I}_{k}\left(x\left(t_{k}\right)\right)=I_{k}\left(m_{\alpha_{2}, \beta_{2}}\left(t_{k}, x\left(t_{k}\right)\right)\right), \tilde{J}_{k}\left(x\left(t_{k}\right), x^{\prime}\left(t_{k}\right)\right)=J_{k}\left(m_{\alpha_{2}, \beta_{2}}\left(t_{k}, x\left(t_{k}\right)\right), n\left(t_{k}, x^{\prime}\left(t_{k}\right)\right)\right)$. Now define the following operator:

$$
\begin{aligned}
(W x)(t)= & \int_{0}^{1} H(t, s) w\left(s, x(s), \frac{d}{d s} m_{\alpha_{2}, \beta_{2}}(s, x)\right) d s \\
& +\sum_{0<t_{k}<t}\left[\tilde{I}_{k}\left(x\left(t_{k}\right)\right)+\left(t-t_{k}\right) \tilde{J}_{k}\left(x\left(t_{k}\right), x^{\prime}\left(t_{k}\right)\right)\right] \\
& -t \sum_{k=1}^{p}\left[\tilde{I}_{k}\left(x\left(t_{k}\right)\right)+\left(1-t_{k}\right) \tilde{J}_{k}\left(x\left(t_{k}\right), x^{\prime}\left(t_{k}\right)\right)\right] \\
& +\frac{1}{1-\mu} \int_{0}^{1} \sum_{0<t_{k}<s}\left[\tilde{I}_{k}\left(x\left(t_{k}\right)\right)+\left(s-t_{k}\right) \tilde{J}_{k}\left(x\left(t_{k}\right), x^{\prime}\left(t_{k}\right)\right)\right] d s \\
& -\frac{v}{1-\mu} \sum_{k=1}^{p}\left[\tilde{I}_{k}\left(x\left(t_{k}\right)\right)+\left(1-t_{k}\right) \tilde{J}_{k}\left(x\left(t_{k}\right), x^{\prime}\left(t_{k}\right)\right)\right] .
\end{aligned}
$$

Again, it is easy to check that $x$ is a solution of (3.3) if $x \in \Omega_{\alpha_{2}}$ and $W x=x$ (note that $W$ : $E \rightarrow E$ is compact). Thus, $\operatorname{deg}\left(I-\bar{T}, \Omega_{\alpha_{2}}, 0\right)=0$. Moreover, it is easy to see that $W(\bar{\Omega}) \subset \Omega$. By assumptions (iv) and (v), there are no solutions in $\partial \Omega^{\beta_{1}} \cup \partial \Omega_{\alpha_{2}}$. So,

$$
\operatorname{deg}\left(I-\bar{T}, \Omega_{\alpha_{2}}, 0\right)=\operatorname{deg}\left(I-W, \Omega \backslash \bar{\Omega}_{\alpha_{2}}, 0\right)+\operatorname{deg}\left(I-W, \Omega_{\alpha_{2}}, 0\right)=\operatorname{deg}(I-W, \Omega, 0)=1
$$

Thus there are three solutions, as required. The proof is complete.

Theorem 3.3 Suppose that conditions $\left(\mathrm{H}_{1}\right)-\left(\mathrm{H}_{3}\right)$ hold. Assume that there exist two lower solutions $\alpha_{1}$ and $\alpha_{2}$ and two upper solutions $\beta_{1}$ and $\beta_{2}$ for problem (1.1), satisfying

(i) $\alpha_{1} \leq \alpha_{2} \leq \beta_{2}$;

(ii) $\alpha_{1} \leq \beta_{1} \leq \beta_{2}$;

(iii) $\alpha_{2} \not \leq \beta_{1}$;

(iv) there exists $0<\bar{\epsilon}<\min _{t \in[0,1]}\left\{\alpha_{2}(t)-\alpha_{1}(t), \beta_{2}(t)-\beta_{1}(t)\right\}$ such that all $\epsilon \in(0, \bar{\epsilon}]$, the functions $\alpha_{2}-\epsilon$ and $\beta_{1}+\epsilon$ are, respectively, lower and upper solutions of (1.1);

(v) $\alpha_{2}-\bar{\epsilon} \not \beta_{1}+\bar{\epsilon}$.

Iff satisfies the Nagumo condition with respect to $\alpha_{1}, \beta_{2}$, then problem (1.1) has at least three solutions $x_{1}, x_{2}$, and $x_{3}$ satisfying

$$
\alpha_{1} \leq x_{1} \leq \beta_{1}, \quad \alpha_{2} \leq x_{2} \leq \beta_{2}, \quad x_{3} \not \leq \beta_{1}, \quad x_{3} \nsucceq \alpha_{2} .
$$


Proof In the proof of Theorem 3.3, define

$$
\begin{aligned}
& \Omega_{\alpha_{2}}=\left\{u \in \Omega: u>\alpha_{2}-\bar{\epsilon} \text { on }(0,1)\right\}, \\
& \Omega_{\beta_{1}}=\left\{u \in \Omega: u<\beta_{1}+\bar{\epsilon} \text { on }(0,1)\right\},
\end{aligned}
$$

where $\Omega$ is defined in Theorem 3.2.

\section{Competing interests}

The authors declare that they have no competing interests.

\section{Authors' contributions}

The authors declare that the study was realized in collaboration with the same responsibility. All authors read and approved the final manuscript.

\section{Acknowledgements}

The work is supported by Beijing Higher Education Young Elite Teacher Project (Project No. YETP0322) and Chinese Universities Scientific Fund (Project No. 2013QJ004).

Received: 7 May 2014 Accepted: 1 July 2014 Published: 22 Jul 2014

\section{References}

1. Lakshmikantham, V, Bainov, DD, Simeonov, PS: Theory of Impulsive Differential Equations. Series in Modern Applied Mathematics, vol. 6. World Scientific, Singapore (1989)

2. Bainov, DD, Simeonov, PS: Systems with Impulse Effect. Ellis Horwood Series: Mathematics and Its Applications. Ellis Horwood, Chichester (1989)

3. Samoilenko, AM, Perestyuk, NA: Impulsive Differential Equations. World Scientific Series on Nonlinear Science: Series A: Monographs and Treatises, vol. 14. World Scientific, Singapore (1995)

4. Agarwal, RP, O'Regan, D: Multiple nonnegative solutions for second order impulsive differential equations. Appl. Math. Comput. 114, 51-59 (2000)

5. Ding, W, Han, M: Periodic boundary value problem for the second order impulsive functional differential equations. Appl. Math. Comput. 155, 709-726 (2004)

6. Lee, EK, Lee, YH: Multiple positive solutions of singular two point boundary value problems for second order impulsive differential equations. Appl. Math. Comput. 158, 745-759 (2004)

7. Gallardo, JM: Second order differential operators with integral boundary conditions and generation of semigroups. Rocky Mt. J. Math. 30, 1265-1292 (2000)

8. Karakostas, GL, Tsamatos, PC: Multiple positive solutions of some Fredholm integral equations arisen from nonlocal boundary-value problems. Electron. J. Differ. Equ. 30, 1-17 (2002)

9. Lomtatidze, A, Malaguti, L: On a nonlocal boundary-value problems for second order nonlinear singular differential equations. Georgian Math. J. 7, 133-154 (2000)

10. Corduneanu, C: Integral Equations and Applications. Cambridge University Press, Cambridge (1991)

11. Agarwal, RP, O'Regan, D: Infinite Interval Problems for Differential, Difference and Integral Equations. Kluwer Academic, Dordrecht (2001)

12. Hao, X, Liu, L, Wu, Y: Positive solutions for second order impulsive differential equations with integral boundary conditions. Commun. Nonlinear Sci. Numer. Simul. 16, 101-111 (2011)

13. Zhang, $X$, Feng, M, Ge, W: Existence of solutions of boundary value problems with integral boundary conditions for second-order impulsive integro-differential equations in Banach spaces. J. Comput. Appl. Math. 233, 1915-1926 (2010)

14. Ding, W, Wang, Y: New result for a class of impulsive differential equation with integral boundary conditions. Commun. Nonlinear Sci. Numer. Simul. 18, 1095-1105 (2013)

15. Cabada, A, Pouso, RL: Existence results for the problem $\left(\phi\left(u^{\prime}\right)\right)^{\prime}=f\left(t, u, u^{\prime}\right)$ with nonlinear boundary conditions. Nonlinear Anal. 35, 221-231 (1999)

16. Lee, Y, Liu, X: Study of singular boundary value problems for second order impulsive differential equations. J. Math. Anal. Appl. 331, 159-176 (2007)

17. Shen, J, Wang, W: Impulsive boundary value problems with nonlinear boundary conditions. Nonlinear Anal. 69 4055-4062 (2008)

18. Wang, MX, Cabada, A, Nieto, JJ: Monotone method for nonlinear second order periodic boundary value problems with Carathéodory functions. Ann. Pol. Math. 58, 221-235 (1993)

10.1186/1687-1847-2014-183

Cite this article as: Pang et al.: The method of upper and lower solutions to impulsive differential equations with integral boundary conditions. Advances in Difference Equations 2014, 2014:183 